\title{
Uncollided Flux Implementation for Discrete Ordinates Radiation Transport Solutions in Rattlesnake
}

\author{
Logan Harbour ${ }^{1}$, Jean Ragusa ${ }^{1}$, Yaqi Wang ${ }^{2}$, Sebastian Schunert ${ }^{2}$, \\ Derek Gaston ${ }^{2}$, Mark DeHart ${ }^{2}$ \\ ${ }^{1}$ Department of Nuclear Engineering \\ Texas A\&M University \\ College Station, TX 77840 \\ ${ }^{2}$ Idaho National Laboratory \\ P.O. Box 1625 \\ Idaho Falls, ID 83415
}

August 2017

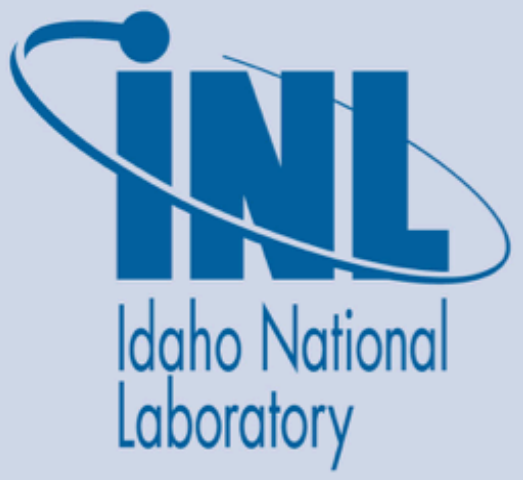

INL is a U.S. Department of Energy National Laboratory operated by Battelle Energy Alliance 


\section{DISCLAIMER}

This information was prepared as an account of work sponsored by an agency of the U.S. Government. Neither the U.S. Government nor any agency thereof, nor any of their employees, makes any warranty, expressed or implied, or assumes any legal liability or responsibility for the accuracy, completeness, or usefulness, of any information, apparatus, product, or process disclosed, or represents that its use would not infringe privately owned rights. References herein to any specific commercial product, process, or service by trade name, trade mark, manufacturer, or otherwise, does not necessarily constitute or imply its endorsement, recommendation, or favoring by the U.S. Government or any agency thereof. The views and opinions of authors expressed herein do not necessarily state or reflect those of the U.S. Government or any agency thereof. 


\section{Uncollided Flux Implementation for Discrete Ordinates Radiation Transport Solutions in Rattlesnake}

Logan Harbour, Jean Ragusa, Yaqi Wang, Sebastian Schunert, Derek Gaston, Mark DeHart

August 2017

Idaho National Laboratory

P.O. Box 1625

Idaho Falls, Idaho 83415

http://www.inl.gov

Prepared for the

U.S. Department of Energy

Office of Nuclear Energy

Under DOE Idaho Operations Office

Contract DE-AC07-05ID14517 


\section{Contents}

$\begin{array}{lll}1 & \text { Introduction } & 1\end{array}$

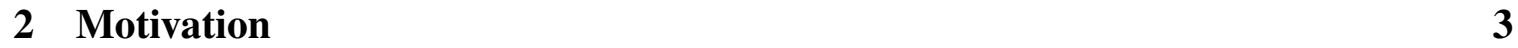

3 Theory 5

$3.1 \quad$ First and Last-Collision Source Treatments . . . . . . . . . . . . . . . . 5

3.2 Uncollided flux computation . . . . . . . . . . . . . . 6

3.2.1 Target cell $\neq$ source cell $\left(K \neq K^{\prime}\right) \ldots \ldots \ldots$. . . . . . 6

3.2.2 Target cell $=$ source cell $\left(K=K^{\prime}\right) \ldots \ldots \ldots$. . . . . . . 7

$3.2 .3 \quad$ Higher-order spatial moments . . . . . . . . . . . . . . . . . . 8

3.2.4 Higher-order angular moments . . . . . . . . . . . . . . 9

\begin{tabular}{lll}
\hline Implementation & 11
\end{tabular}

4.1 Algorithm . . . . . . . . . . . . . . . . . . 11

5 Verification 13

5.1 Point Source to Volumetric Target . . . . . . . . . . . . . . . . . . . 13

5.2 Volumetric Source to Volumetric Targets . . . . . . . . . . . . . . . 15

5.3 Kobayashi Benchmark . . . . . . . . . . . . . . . . . 17

6 Future Work $\quad 21$

6.1 Ray-Tracing Functionality . . . . . . . . . . . . . . . . 21

6.1 .1 Tracing to target surfaces and points . . . . . . . . . . . . 21

6.1 .2 Reflective boundary conditions . . . . . . . . . . . . . . 21

6.2 Methods development . . . . . . . . . . . . . . . . . . 22

6.2 .1 First and last-collided Source. . . . . . . . . . . . . . . . . . 22

6.2 .2 Spatial and angular moments . . . . . . . . . . . . 22

6.3 Conclusions . . . . . . . . . . . . . . . . . 23 


\section{List of Figures}

$1 \quad$ Plan and elevation views of a cross-section of the reactor, showing the relative orientation of the core, a test item, reactor structural materials, and components of the $1.2 \mathrm{~m}$ collimator hodoscope at TREAT [6]. . . . . . . . 4

2 Comparison of relative errors in the integrated uncollided flux for two elements (where an element is defined by its lower corner) in a homogeneous medium with varying absorption. . . . . . . . . . . . . . . . 14

3 Comparison of relative errors in the integrated uncollided flux in each element in a void medium with 8 Gaussian quadrature points per element. . . . 14

4 Comparison of relative errors in the integrated uncollided flux in each element in an absorbing medium $\left(\Sigma_{t}=0.5 \mathrm{~cm}\right)$ with 8 Gaussian quadrature points per element. ................. 15

5 Comparison of relative errors in the integrated uncollided flux for two elements with a volumetric source and target. Element $x=4$ is the element defined by the corners $(4,0,0)$ and $(5,1,1)$ and element $x=8$ is the element defined by the corners $(8,0,0)$ and $(9,1,1) . \Sigma_{t}^{s}$ is the source cross section and $\Sigma_{t}^{t}$ is the target cross section. $\ldots \ldots \ldots \ldots \ldots$

$6 \quad$ The geometry for the Kobayashi benchmark problem ii. [7] . . . . . . . . . 17

7 The cell-averaged uncollided flux result for the Kobayashi benchmark problem ii in a pure absorber. Viewed is the duct extending directly from the source elements. The contour lines are evenly spaced between 1 and $10^{-3}$ (log scale). The axes are shifted $10 \mathrm{~cm}$ in every direction compared to the benchmark geometries in order to account for the reflecting boundaries. . . 18

8 The cell-averaged uncollided flux result for the Kobayashi benchmark problem ii in a pure absorber. Viewed is the top duct plane extending towards the rear $y-z$ face. The contour lines are evenly spaced between $10^{-4}$ and $10^{-6}$ (log scale). The axes are shifted $10 \mathrm{~cm}$ in every direction compared to the benchmark geometries in order to account for the reflecting boundaries. 19 


\section{List of Tables}

1 Point source to volumetric target results in void and a uniform absorber for two elements. . . . . . . . . . . . . . . . . . . 13

$2 \quad$ Volumetric source to volumetric target results for the element defined by the corners $(4,0,0)$ and $(5,1,1)$ and the element defined by the corners $(8,0,0)$ and $(9,1,1) . \ldots \ldots \ldots \ldots$ 


\section{Introduction}

Operating from February 1959 until April 1994, the Transient Reactor Test Facility (TREAT) at Idaho National Laboratory (INL) was specifically built to conduct transient reactor tests where the test material is subjected to neutron pulses that can simulate conditions ranging from mild upsets to severe reactor accidents. The reactor was constructed to test fast reactor fuels, but has also been used for light water reactor fuel testing as well as other exotic special purpose fuels. [1, 2] TREAT is an air-cooled, thermal-spectrum test facility designed to evaluate reactor fuels and structural materials under simulated nuclear excursions and transient power/cooling mismatch situations in a nuclear reactor [3]. Such testing involves placing fuel or material into the TREAT core and subjecting it to short bursts of intense, high-power neutron radiation. After the experiment is completed, the fuel or material is analyzed to determine the effects of the power burst. The resulting information is then used to guide the development and improvement of advanced nuclear fuel designs, and to validate computer models of fuel and core behavior required for U.S. Nuclear Regulatory Commission (NRC) evaluation of nuclear power reactor design and safety evaluations [2].

Historical methods used for pre-transient calculations were very approximate low order methods that required a number of reduced and full power tests to obtain correction factors to improve the prediction of the full power transient prior to the test itself. The use of modern modeling and simulation $(\mathrm{M} \& \mathrm{~S})$ tools, capturing the multi-physics nature of TREAT operation and experimentation, has the potential for reducing the number of low and high power calibration tests needed prior to full power operations for a transient experiment. This would result in significant operational efficiency with corresponding cost savings, and would also lay the groundwork for improved fidelity in experiment design. Hence, INL is engaged in efforts to develop full multi-physics modeling capabilities to predict core transient behavior (power excursions with thermal feedback) and its coupling with experimental configurations, supported by the Nuclear Energy Advanced Modeling and Simulation (NEAMS) program within the U.S. Department of Energy Office of Nuclear Energy [4].

Transient testing involves placement of fuel samples into the core of specialized test reactors that are capable of performing a range of transient maneuvers to simulate accident conditions that require the reactor produce short bursts of hight neutron flux and gamma radiation. Testing fuel in a high flux environment is used to generate high power energy deposition in test fuel samples, accident-simulation conditions is a key step in licensing nuclear fuels for use in existing and future nuclear power plants. Transient testing of nuclear fuels is needed to develop and prove the safety basis for advanced reactors and fuels. In addition, modern fuel development and design increasingly relies on modeling and simulation 
efforts that must be informed and validated using specially designed material performance separate effects studies. These studies will require experimental facilities that are able to support variable scale, highly instrumented tests providing data that have appropriate spatial and temporal resolution. Finally, there are efforts now underway to develop advanced light water reactor (LWR) fuels with enhanced performance and accident tolerance. These advanced reactor designs will also require new fuel types. These new fuels need to be tested in a controlled environment in order to learn how they respond to accident conditions. For these applications, transient reactor testing is needed to help design fuels with improved performance.

Early in TREAT operations, a fast neutron hodoscope was added to TREAT to providing a real-time imaging technology. A hodoscope is an instrument used to detect particles emitted from a source and determine their trajectories. Hodoscopes are generally constructed of several segments of filter, collimators and detectors; the combination of these segments is then used to infer where the particle passed through hodoscope by the location at which the particle is detected. A fast-neutron hodoscope is used to detect fast neutrons emitted as a result of fissions in a nuclear fuel sample. The TREAT hodoscope system was developed to provide a imaging system to view fuel motion and failure during rapid power excursions, albeit with a limited degree of spatial resolution. The hodoscope concept was proposed in 1963 and tests performed in 1964 showed a high signal-to-noise ratio for fast-neutron detection. The hodoscope was first operational within TREAT by 1965, although it has gone through a number of upgrades over its existence, and additional, more high-fidelity upgrades are anticipated in the next few years after restart.

The fast-neutron hodoscope at TREAT has been used to detect fuel motion within test samples inserted in the center of the reactor core. The hodoscope system was built to support nuclear fuel performance testing by providing a mechanism to detect when fuel motion is induced under simulated accident conditions. Optical detection methods were used in the past by enclosing test capsules within a quartz vessel; however, quartz does not have the strength necessary to maintain structural integrity for experiments performed in pressures representative of reactor systems. Hence optical methods cannot be used with high pressure containment environments due to opacity of pressure vessel walls, as well as other experimental apparati. However, those materials are generally transparent to fast neutrons generated withing the test fuel specimen(s).

The current TREAT hodoscope system includes components necessary to collect, store and process data providing time and spatial resolution of fuel motion prior to, during and after a transient experiment. The hodoscope uses collimators and two sets of detectors located outside the reactor core for fast neutron measurements. Thus, it relies on fast neutrons 
born in the test sample(s) to travel significant distances (approximately $3 \mathrm{~m}$ ) through air to reach the detector pairs. The TREAT hodoscope configuration is illustrated in Fig. 1. As seen by the hodoscope, the planar cross sectional area at the location of test fuel samples measures $5.7 \mathrm{~cm}$ in width by $52 \mathrm{~cm}$ in height, with 10 columns and 36 rows of slots in a steel collimator for a total of 360 slots; however, a spatial resolution of fuel motion as small as 0.25 by $8 \mathrm{~mm}$ can be achieved. The exits of the collimator slots are aligned with the two back-to-back neutron detectors. The first is a methane gas-filled proportional counter adapted from a design proven for the hodoscope used at the CABRI transient facility; the second is a proton-recoil scintillator that consists of a thin film of $\mathrm{ZnS}$ applied to lucite, which together form the button, known as a Hornyak button. Scintillations within the buttons are amplified in photomultiplier tubes. Data acquisition systems collect data in real time for each detector pair at each collimator slot. The collimator and detectors are external to the reactor.

\section{Motivation}

Simulation of neutron streaming over long distances in optically thin narrow pipes surrounded by optically thicker materials that can absorb and/or scatter neutrons presents a simulation challenge to most neutron transport methods. Without sophisticated variance reduction techniques, this problem can be intractable for Monte Carlo techniques. Deterministic methods are often limited due to large angular discretization errors, this phenomenon is commonly known as ray effects. The uncollided component of the angular flux solution is the most anisotropic part and it can be difficult for discrete ordinate methods to accurately represent. The number of discrete angles required to overcome these ray effects is prohibitively large for the hodoscope configuration. Hence, other approaches must be taken to better simulate neutron streaming within the hodoscope collimator slots.

Note that the approach we are proposing for the hodoscope is not necessary in order to model the TREAT core itself. Other streaming effects are present within the core itself, notably within air gaps between between fuel elements and between the core and its surrounding permanent reflector; these streaming effects are treated differently[4]. The in present work we are focused on correction for the inability of a discrete ordinates approach to yield accurate uncollided fluxes in the fast-neutron hodoscope due to ray effects.

Angular discretization techniques $\left(S_{N}\right.$ and $\left.P_{N}\right)$ for the transport equation are notoriously poor at accurately capturing streaming effects. However, it has long been recognized that 
an analytical or semi-analytical treatment of the uncollided flux, coupled with a discrete ordinate treatment of the collided flux, can yield dramatic improvements in accuracy and computational efficiency. In this report, we present an algorithm for semi-analytical calculation of the uncollided flux. The algorithm seeks to compute:

1. The exiting uncollided flux for a set of points located in a volume of interest (e.g., detector regions) or on surfaces of interest (e.g., detector surfaces). The uncollided angular flux is reported for each source point (e.g., points in a testing apparatus such as the Multi-SERTTA test vessel[5]). The direction of flight is from the source point to the target (surface or volume) point. The source points are chosen to be spatial quadrature points that integrate the source volume (or source surface).

2. Spatial and angular distributions of the uncollided flux in every cell in the problem. These provide all of the information necessary to form the first-collision source in each cell.

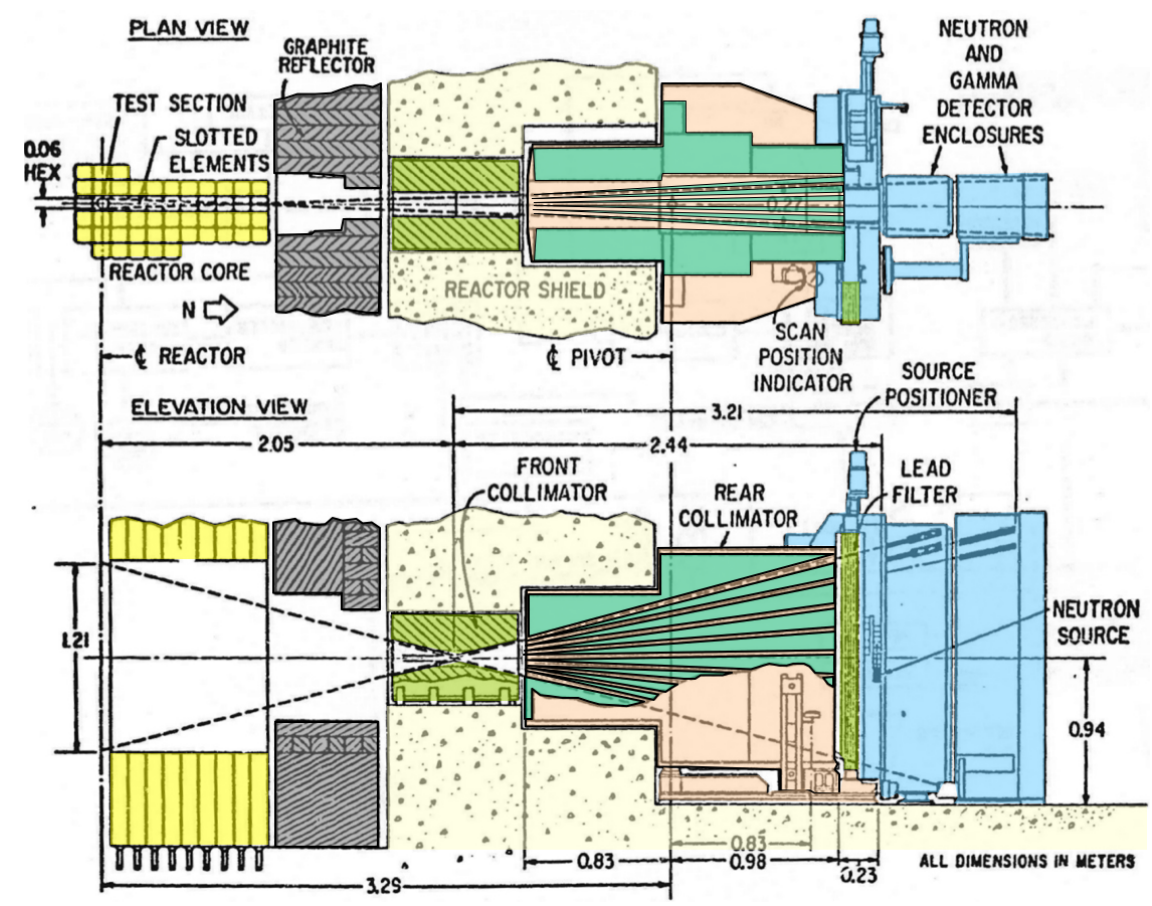

Figure 1: Plan and elevation views of a cross-section of the reactor, showing the relative orientation of the core, a test item, reactor structural materials, and components of the 1.2 m collimator hodoscope at TREAT [6]. 


\section{Theory}

\subsection{First and Last-Collision Source Treatments}

Using operator notation, the transport problem can be viewed as

$$
L \psi=H \psi+Q,
$$

where $L$ is the streaming+collision operator $\left(\boldsymbol{\Omega} \cdot \boldsymbol{\nabla}+\sigma_{t}\right), H$ is the scattering operator, and $Q$ is an extraneous source. The $P_{N}$ method requires a high degree $N$ to capture anisotropic angular flux distributions and the $S_{N}$ method suffers from ray effects due to the angular quadratures only being rotationally invariant by octant.

However, the $L$ operator can be inverted semi-analytically with great accuracy through raytracing in arbitrary geometries. Hence, the idea of decomposing the flux into collided and uncollided components as $\psi=\psi^{u}+\psi^{c}$. The uncollided component is obtained from

$$
L^{R T} \psi^{u, R T}=Q,
$$

where the superscript $R T$ denotes ray-tracing. Using the uncollided flux, a first-collided scattering source $H \psi^{u, R T}$ is created for the collided equation as

$$
L^{S N} \psi^{c}=H \psi^{c}+H \psi^{u, R T},
$$

where the superscript $S N$ denotes the $S_{N}$ solve. Typically, a source iteration process is then employed to solve the above equation:

$$
L^{S N} \psi^{c, \ell+1}=H \psi^{c, \ell}+H \psi u, R T .
$$

Upon convergence (superscript $\infty$ ), the total angular flux is obtained by summation $\psi=$ $\psi^{u, R T}+\psi^{c, \infty}$.

If $\psi^{c}$ contributes significantly to the desired quantities of interest (e.g., detector reaction rate) and still suffers from ray effects, we can further refine the methodology by using the collided $S N$ solution as a means to compute the scattering source due to particles having scattered more than once, $H \psi^{c, \infty}$, and one additional ray-tracing is performed toward the detector location. This is called the last-collision source process and it also uses ray-tracing and delivers the full angular flux $\psi^{R T}=\psi^{u, R T}+\psi^{c, R T}$ as

$$
L^{R T} \psi^{R T}=H \psi^{c, \infty}+H \psi^{u, r t}+Q,
$$


or, one can just solve for the ray-tracing collided part (because we already have the raytracing uncollided part from $\left.L^{R T} \psi^{u, R T}=Q\right)$ :

$$
L^{R T} \psi^{c, R T}=H \psi^{c, \infty}+H \psi^{u, R T} .
$$

\subsection{Uncollided flux computation}

First, we propose a method to compute the uncollided flux for any target cells, $K$ (where $K \in(\mathscr{D} \backslash \mathscr{S})$ ) that are different than the source cell, $\left.K^{\prime} \in \mathscr{S}\right)$. With this notation, $\mathscr{D}$ describes the entire domain and $\mathscr{S}$ describes the subset of the domain that contains the source(s). Note that by superposition principle, we can deal with all source cells simultaneously (the ray tracing process due to multiple source cells is embarrassingly parallel). However, for clarity, the method is presented for one source at a time and the uncollided flux is computed in all cells due to that one source (we distinguish between the target cell containing that source, $K=K^{\prime}$, or not, $K \neq K^{\prime}$ ).

\subsubsection{Target cell $\neq$ source cell $\left(K \neq K^{\prime}\right)$}

The exact uncollided scalar flux at a quadrature point $q$ in a target cell $K$ as a result of a volumetric source cell $K^{\prime}$ is

$$
\phi^{\text {exact }}\left(\mathbf{r}_{q}\right)=\int_{K^{\prime}} d \mathbf{r} \frac{\exp \left(-\tau\left(\mathbf{r}, \mathbf{r}_{q}\right)\right)}{R_{q}^{2}(\mathbf{r})} S\left(\mathbf{r}, \boldsymbol{\Omega}_{q}(\mathbf{r})\right)
$$

where $\tau\left(\mathbf{r}, \mathbf{r}_{q}\right)$ is the optical thickness from a source point at $\mathbf{r}$ to a target quadrature point at $\mathbf{r}_{q}, R_{q}(\mathbf{r})=\left|\mathbf{r}-\mathbf{r}_{q}\right|, \boldsymbol{\Omega}_{q}(\mathbf{r})$ is the direction from source position $\mathbf{r}$ to target quadrature point $\mathbf{r}_{q}$, and $S$ has units $\mathrm{n} / \mathrm{cm}^{3} \cdot \mathrm{s} \cdot \mathrm{str}$.

Introducing spatial quadrature $q^{\prime}$ in the source cell $K^{\prime}$ with weight $w_{q^{\prime}}$ we have

$$
\phi\left(\mathbf{r}_{q}\right)=\sum_{q^{\prime}} w_{q^{\prime}} \frac{\exp \left(-\tau\left(\mathbf{r}_{q^{\prime}}, \mathbf{r}_{q}\right)\right)}{R_{q^{\prime} q}^{2}(\mathbf{r})} S\left(\mathbf{r}_{q^{\prime}}, \boldsymbol{\Omega}_{q^{\prime} q}\right),
$$

where $R_{q^{\prime} q}$ is the distance from source quadrature point $\mathbf{r}_{q^{\prime}}$ to target quadrature point $\mathbf{r}_{q}$, and $\boldsymbol{\Omega}_{q^{\prime} q}$ is the direction from source quadrature point $\mathbf{r}_{q^{\prime}}$ to target quadrature point $\mathbf{r}_{q}$. 
Lastly, introducing spatial quadrature $q$ in the target cell with weight $w_{q}$, we then have the cell-average uncollided scalar flux in cell $K$ (as a result of source cell $K^{\prime}$ ) as

$$
\bar{\phi}_{K}^{K^{\prime}}=\frac{1}{V_{K}} \sum_{q} w_{q} \sum_{q^{\prime}} w_{q}^{\prime} \frac{\exp \left(-\tau\left(\mathbf{r}_{q^{\prime}}, \mathbf{r}_{q}\right)\right)}{R_{q^{\prime} q}^{2}(\mathbf{r})} S\left(\mathbf{r}_{q^{\prime}}, \boldsymbol{\Omega}_{q^{\prime} q}\right)
$$

where $V_{K}$ is the volume of target cell $K$.

\subsection{2 $\quad$ Target cell $=$ source cell $\left(K=K^{\prime}\right)$}

With the above, the uncollided angular flux was computed using Green's function which contains a $1 / R^{2}$ term. Hence, computing the uncollided flux inside the source cell could be problematic. It could potentially be inaccurate in the neighboring cells of the source cells as well. To combat this, we use the following approach.

The cell-averaged scalar flux in a given cell $K$ due to a source in the same cell is

$$
\bar{\phi}_{K}^{K}=\frac{1}{V_{K}} \int_{K} d \mathbf{r} \int_{K^{\prime}} d \mathbf{r}^{\prime} \frac{\exp \left(-\tau\left(\mathbf{r}, \mathbf{r}^{\prime}\right)\right)}{R^{2}} S\left(\mathbf{r}^{\prime}, \mathbf{\Omega}_{R}\right),
$$

where $\mathbf{R}=\mathbf{r}-\mathbf{r}^{\prime}, R=\|\mathbf{R}\|$, and $\boldsymbol{\Omega}=\mathbf{R} / R$. When $K=K^{\prime}$, we will eliminate the $1 / R^{2}$ term by introducing the change of variable $\mathbf{r}^{\prime}=\mathbf{r}-\mathbf{R}=\mathbf{r}-R \boldsymbol{\Omega}$, so that $d \mathbf{r}^{\prime}=d \mathbf{r}=R^{2} d R d \Omega$ to obtain

$$
\bar{\phi}_{K}^{K}=\frac{1}{V_{K}} \int_{K} d \mathbf{r} \int_{4 \pi} d \boldsymbol{\Omega} \int_{R_{-}(\mathbf{r}, \boldsymbol{\Omega})}^{R_{+}(\mathbf{r}, \boldsymbol{\Omega})} d R \exp \left(-\tau\left(\mathbf{r}, \mathbf{r}^{\prime}\right)\right) S\left(\mathbf{r}^{\prime}, \mathbf{\Omega}\right),
$$

where $R_{ \pm}$is the distance from $\mathbf{r}$ to the first $\left(R_{-}\right)$or second $R_{+}$intersections of the back trajectory at $\mathbf{r}$ in the direction $\boldsymbol{\Omega}$ with the surface of $K^{\prime}$ (assuming the later is convex). We then require a quadrature for $\boldsymbol{\Omega}$ (for example, the one used for the $S_{N}$ discrete ordinate approximation) and a quadrature for $\mathbf{r}$ in $K$.

Noting that $R_{-}(\mathbf{r}, \boldsymbol{\Omega})=0$ and $\mathbf{r}^{\prime}=\mathbf{r}-R \boldsymbol{\Omega}$, we then have the exact cell-averaged uncollided scalar flux (as the result of source cell $K^{\prime}=$ target cell $K$, zeroth spatial and angular moments) as

$$
\bar{\phi}_{K}^{K}=\frac{1}{V_{K}} \int_{K} d \mathbf{r} \int_{4 \pi} d \boldsymbol{\Omega} \int_{0}^{R_{+}(\mathbf{r}, \boldsymbol{\Omega})} d R \exp (-\tau(\mathbf{r}, \mathbf{r}-R \boldsymbol{\Omega})) S(\mathbf{r}-R \boldsymbol{\Omega}, \boldsymbol{\Omega}) .
$$

Introducing 1D quadrature $q$ with weights $w_{q}$ along the ray direction $\boldsymbol{\Omega}$

$$
\bar{\phi}_{K}^{K}=\frac{1}{V_{K}} \int_{K} d \mathbf{r} \int_{4 \pi} d \Omega \sum_{q} w_{q} \exp \left(-\tau\left(\mathbf{r}, \mathbf{r}-R_{q} \boldsymbol{\Omega}\right)\right) S\left(\mathbf{r}-R_{q} \boldsymbol{\Omega}, \boldsymbol{\Omega}\right),
$$


introducing angular quadrature $d$ with solid angle weights $\omega_{d}$

$$
\bar{\phi}_{K}^{K}=\frac{1}{V_{K}} \int_{K} d \mathbf{r} \sum_{d} \omega_{d} \sum_{q} w_{q} \exp \left(-\tau\left(\mathbf{r}, \mathbf{r}-R_{q} \boldsymbol{\Omega}_{d}\right)\right) S\left(\mathbf{r}-R_{q} \boldsymbol{\Omega}, \boldsymbol{\Omega}_{d}\right)
$$

and lastly introducing 3D quadrature $j$ with volumetric weights $w_{j}$ we have

$$
\bar{\phi}_{K}^{K}=\frac{1}{V_{K}} \sum_{j} w_{j} \sum_{d} \omega_{d} \sum_{q} w_{q} \exp \left(-\tau\left(\mathbf{r}_{j}, \mathbf{r}_{j}-R_{q} \boldsymbol{\Omega}_{d}\right)\right) S\left(\mathbf{r}_{j}-R_{q} \boldsymbol{\Omega}, \boldsymbol{\Omega}_{d}\right) .
$$

\subsubsection{Higher-order spatial moments}

With the addition of a basis function $b(\mathbf{r})$ in the target cell $K$, the spatial moment of the scalar flux in target cell $K$ due to source cell $K^{\prime}$ is then

$$
\phi_{K, i}^{K^{\prime}}(\mathbf{r})=\int_{K} d \mathbf{r} b_{i}(\mathbf{r}) \int_{K^{\prime}} d \mathbf{r}^{\prime} \frac{\exp \left(-\tau\left(\mathbf{r}^{\prime}, \mathbf{r}\right)\right)}{R^{2}(\mathbf{r})} S\left(\mathbf{r}^{\prime}, \boldsymbol{\Omega}_{R}\right), \quad \forall \mathbf{r} \in K
$$

where $\mathbf{R}=\mathbf{r}^{\prime}-\mathbf{r}, R=\|\mathbf{R}\|$, and $\boldsymbol{\Omega}_{R}=\mathbf{R} / R$. Introducing $3 D$ quadrature $q^{\prime}$ in the source cell $K^{\prime}$ with volumetric weights $w_{q^{\prime}}$ and 3D quadrature $q$ in the target cell $K$ with volumetric weights $w_{q}$ leads to

$$
\phi_{K, i}^{K^{\prime}}(\mathbf{r}) \approx \sum_{q} w_{q} b_{i}\left(\mathbf{r}_{q}\right) \sum_{q^{\prime}} w_{q^{\prime}} \frac{\exp \left(-\tau\left(\mathbf{r}_{q^{\prime}}, \mathbf{r}_{q}\right)\right)}{R_{q^{\prime} q}^{2}(\mathbf{r})} S\left(\mathbf{r}_{q^{\prime}}, \boldsymbol{\Omega}_{q^{\prime} q}\right), \quad \forall \mathbf{r} \in K .
$$

The same method is applied to the target $=$ source cell $\left(K^{\prime}=K\right)$ method as

$$
\phi_{K, i}^{K}(\mathbf{r})=\int_{K} d \mathbf{r} b_{i}(\mathbf{r}) \int_{4 \pi} d \Omega \int_{0}^{R_{+}(\mathbf{r}, \boldsymbol{\Omega})} d R \exp (-\tau(\mathbf{r}, \mathbf{r}-R \boldsymbol{\Omega})) S(\mathbf{r}-R \boldsymbol{\Omega}, \boldsymbol{\Omega}), \quad \forall \mathbf{r} \in K,
$$

and with the same quadratures used in Eq. (2) as

$$
\phi_{K, i}^{K}(\mathbf{r}) \approx \sum_{j} w_{j} b_{i}\left(r_{j}\right) \sum_{d} \omega_{d} \sum_{q} w_{q} \exp \left(-\tau\left(\mathbf{r}_{j}, \mathbf{r}_{j}-R_{q} \boldsymbol{\Omega}_{d}\right)\right) S\left(\mathbf{r}_{j}-R_{q} \boldsymbol{\Omega}, \boldsymbol{\Omega}_{d}\right), \quad \forall \mathbf{r} \in K .
$$




\subsubsection{Higher-order angular moments}

When scattering is anisotropic, one needs a higher angular moment than the scalar flux. The angular moment of the scalar flux in target cell $K$ due to source cell $K^{\prime}$ is

$$
\begin{aligned}
\phi_{K, k}^{K^{\prime}}(\mathbf{r}) & =\int_{K^{\prime}} d \mathbf{r}^{\prime} \int_{4 \pi} d \Omega A_{k}(\boldsymbol{\Omega}) \frac{\exp \left(-\tau\left(\mathbf{r}^{\prime}, \mathbf{r}\right)\right)}{R^{2}(\mathbf{r})} S\left(\mathbf{r}^{\prime}, \boldsymbol{\Omega}\right) \delta_{2}\left(\boldsymbol{\Omega} \cdot \boldsymbol{\Omega}_{R}\right), \quad \forall \mathbf{r} \in K, \\
& =\int_{K^{\prime}} d \mathbf{r}^{\prime} A_{k}\left(\boldsymbol{\Omega}_{R}\right) \frac{\exp \left(-\tau\left(\mathbf{r}^{\prime}, \mathbf{r}\right)\right)}{R^{2}(\mathbf{r})} S\left(\mathbf{r}^{\prime}, \boldsymbol{\Omega}_{R}\right), \quad \forall \mathbf{r} \in K .
\end{aligned}
$$

Again introducing 3D quadrature $q^{\prime}$ in the source cell $K^{\prime}$ we have

$$
\phi_{K, k}^{K^{\prime}}(\mathbf{r}) \approx \sum_{q^{\prime}} w_{q^{\prime}} A_{k}\left(\boldsymbol{\Omega}_{q^{\prime}}(\mathbf{r})\right) \frac{\exp \left(-\tau\left(\mathbf{r}_{q^{\prime}}, \mathbf{r}\right)\right)}{R_{q^{\prime}}^{2}(\mathbf{r})} S\left(\mathbf{r}_{q^{\prime}}, \boldsymbol{\Omega}_{q^{\prime}}(\mathbf{r})\right), \quad \forall \mathbf{r} \in K
$$

where $\boldsymbol{\Omega}_{q^{\prime}}(\mathbf{r})$ is the direction from source quadrature point $\mathbf{r}_{q^{\prime}}$ to $\mathbf{r}$ and $R_{q^{\prime}}(\mathbf{r})$ is the distance from source quadrature point $\mathbf{r}_{q^{\prime}}$ to $\mathbf{r}$. 


\section{Implementation}

The ray_tracing module developed by D. Gaston for MOOSE is currently in development and not presently available in production. Pre-production access was given for the purposes of making the initial implementation of the uncollided flux treatment in Rattlesnake. It provides a straight-forward framework for the tracing of rays in an arbitrary mesh. The user is provided with a method to generate rays and the ability to store solutions along ray segments and at the termination of a ray. For the purposes of this report, only the implementation in Rattlesnake on top of the ray_tracing module is discussed.

The implementation thus far has the ability to determine the element-average uncollided flux as a result of a set of point sources and volumetric sources. This solution can then be stored and utilized as a first-collided source in Rattlesnake. Further goals are discussed later.

The ability to recursively generate, communicate, and propagate rays exists in the ray_tracing module. This method was chosen in order to maximize the parallel efficiency in cases where only localized sources exist in the mesh. When the mesh is partitioned in the case of a distributed mesh, rays can only be started on the processors that contain the portion of the mesh that the sources are in. As a result of this, the remaining processors that do not contain sources sit idle waiting to receive rays. By using a recursive algorithm, sources containing processors are able to both generate and propagate rays out of their local domains in order to provide work for the idle processors. Note the distinction between generation and execution: a generated ray is only a start point, end point, and initialization of data for a single ray, while an executed ray is the propagation of said generated ray.

\subsection{Algorithm}

The general process followed in a distributed mesh is as follows:

1. Each processor stores the local ray targets (portions of the domain where the uncollided flux is desired). This is a list of elements and the points in each element that need to be traced to, in addition to the necessary weight if quadrature is used.

2. The list of local ray targets is serialized and communicated to all other processors so that each source processor has the knowledge of the end points of each ray that it 
spawns.

3. Each processor determines if it contains any sources as provided by the user. If a processor does not contain any sources, it waits to receive and propagate rays. If it contains sources, it generates a ray for each local source point and target point pair and executes the rays. To make these calls recursive, rays are first generated and then executed once a user defined number of rays has been generated. After a chunk of rays has been executed, a source processor will propagate the generated rays in addition to propagating any other rays that have been received by neighboring processors, and then will again continue generation. 


\section{Verification}

\subsection{Point Source to Volumetric Target}

A point source that emits $1 \mathrm{n} / \mathrm{s}$ is located at $(0.5,0.5,0.5)$ in a $10 \times 10 \times 2$ mesh of cuboid elements of width $1 \mathrm{~cm} \times 1 \mathrm{~cm} \times 1 \mathrm{~cm}$. The desired quantity is the integrated uncollided flux in each element (the target elements) except for the element that contains the source (the element with lower vertex $(0,0,0)$ and upper vertex $(1,1,1))$. The total cross section of the medium is then varied and each element evaluated for multiple Gaussian quadratures. The reference solution was evaluated numerically to a relative error tolerance of $1 \times 10^{-12}$. The results for two of the target elements are as seen below in Table 1 and Figure 2.

Table 1: Point source to volumetric target results in void and a uniform absorber for two elements.

\begin{tabular}{cccccc}
\hline $\begin{array}{c}\text { Element } \\
\text { (corners) }\end{array}$ & $\begin{array}{c}\text { Quad pts. } \\
(1 \mathrm{D})\end{array}$ & Solution & Void & \multicolumn{2}{c}{ Uniform absorber $\left(\Sigma_{t}=0.5 \mathrm{~cm}\right)$} \\
& 1 & $3.1830988618 \times 10^{-3}$ & $3.2626694867 \times 10^{-3}$ & $2.6128466569 \times 10^{-4}$ & $2.1467300816 \times 10^{-2}$ \\
& 2 & $3.1933217069 \times 10^{-3}$ & $6.1546384388 \times 10^{-5}$ & $2.6692936268 \times 10^{-4}$ & $3.2744340364 \times 10^{-4}$ \\
$(0,5,0)$, & 3 & $3.1935179346 \times 10^{-3}$ & $1.0078175060 \times 10^{-7}$ & $2.6701654024 \times 10^{-4}$ & $9.5622707194 \times 10^{-7}$ \\
$(1,6,1)$ & 4 & $3.1935182545 \times 10^{-3}$ & $6.0666534586 \times 10^{-10}$ & $2.6701679429 \times 10^{-4}$ & $4.7996283983 \times 10^{-9}$ \\
& 5 & $3.1935182564 \times 10^{-3}$ & $1.0592426617 \times 10^{-12}$ & $2.6701679556 \times 10^{-4}$ & $1.1241697843 \times 10^{-13}$ \\
\cline { 2 - 7 } & Reference & $3.1935182564 \times 10^{-3}$ & & $2.6701679557 \times 10^{-4}$ & \\
\hline & 1 & $1.5915494309 \times 10^{-3}$ & $1.6754904027 \times 10^{-3}$ & $4.6382832411 \times 10^{-5}$ & $1.7853101037 \times 10^{-2}$ \\
$(0,5,1)$, & 3 & $1.5942238602 \times 10^{-3}$ & $2.0875784420 \times 10^{-6}$ & $4.7225741557 \times 10^{-5}$ & $4.6739245577 \times 10^{-6}$ \\
$(1,6,2)$ & 4 & $1.5942205464 \times 10^{-3}$ & $8.9790695168 \times 10^{-9}$ & $4.7225967182 \times 10^{-5}$ & $1.0363500629 \times 10^{-7}$ \\
& 5 & $1.5942205321 \times 10^{-3}$ & $3.1283814836 \times 10^{-15}$ & $4.7225962288 \times 10^{-5}$ & $1.3243756145 \times 10^{-13}$ \\
\hline
\end{tabular}

As the distance between source and target element is increased, the solid angle of the target element as viewed by the source decreases. The solution error then decreases as the source and target distance increases. In order to visualize this effect, the relative errors for each target element in the mesh were plotted as seen below in Figures 3 and 4 . A small target quadrature was utilized in each case in order to represent the error differential as much as possible. 


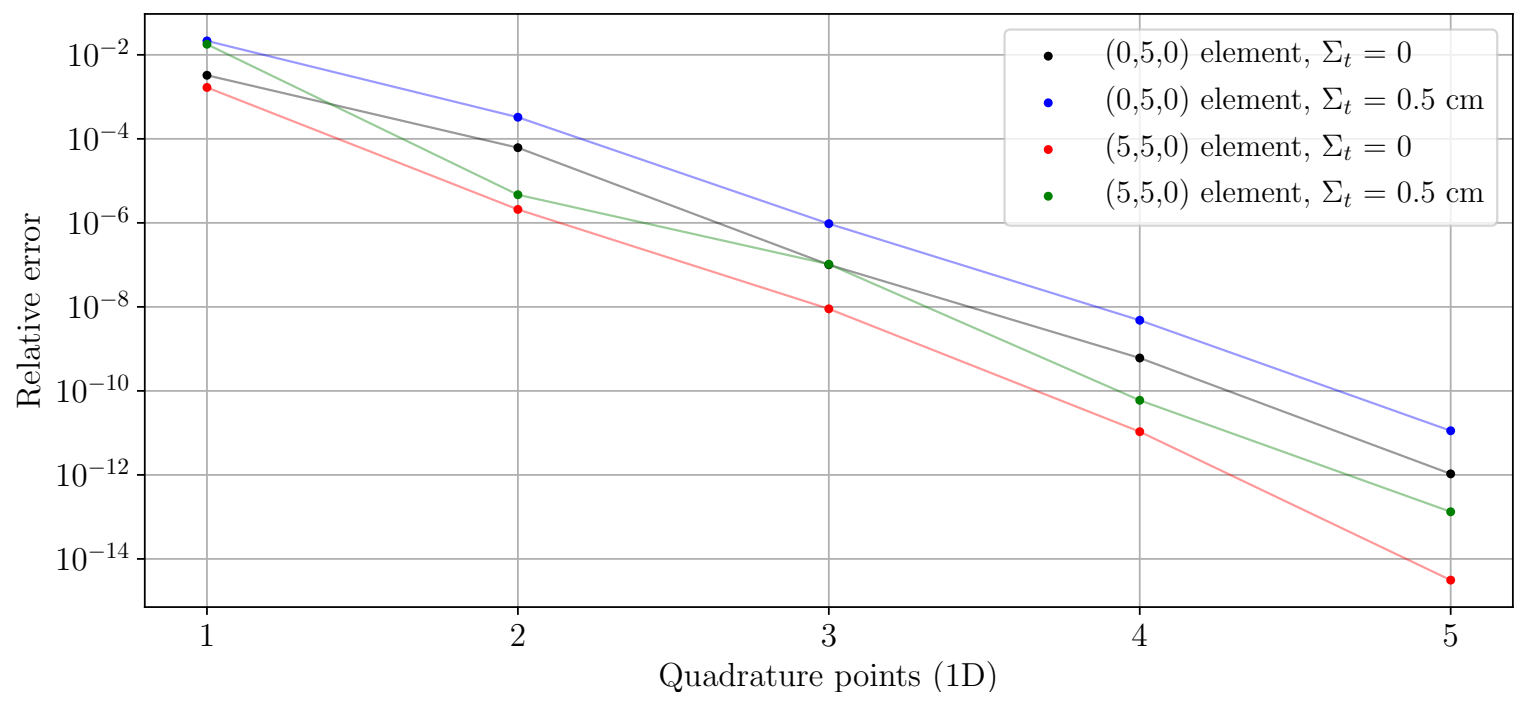

Figure 2: Comparison of relative errors in the integrated uncollided flux for two elements (where an element is defined by its lower corner) in a homogeneous medium with varying absorption.
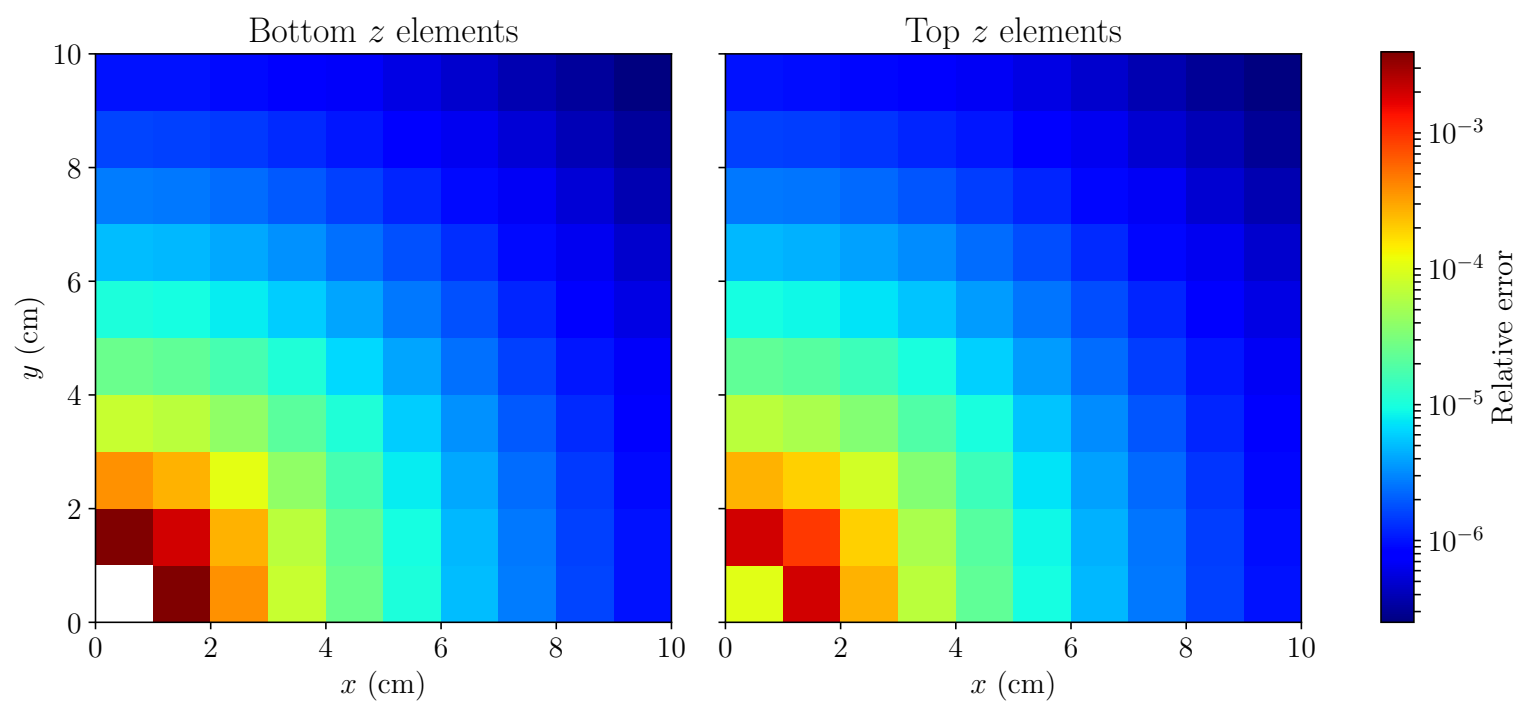

Figure 3: Comparison of relative errors in the integrated uncollided flux in each element in a void medium with 8 Gaussian quadrature points per element. 

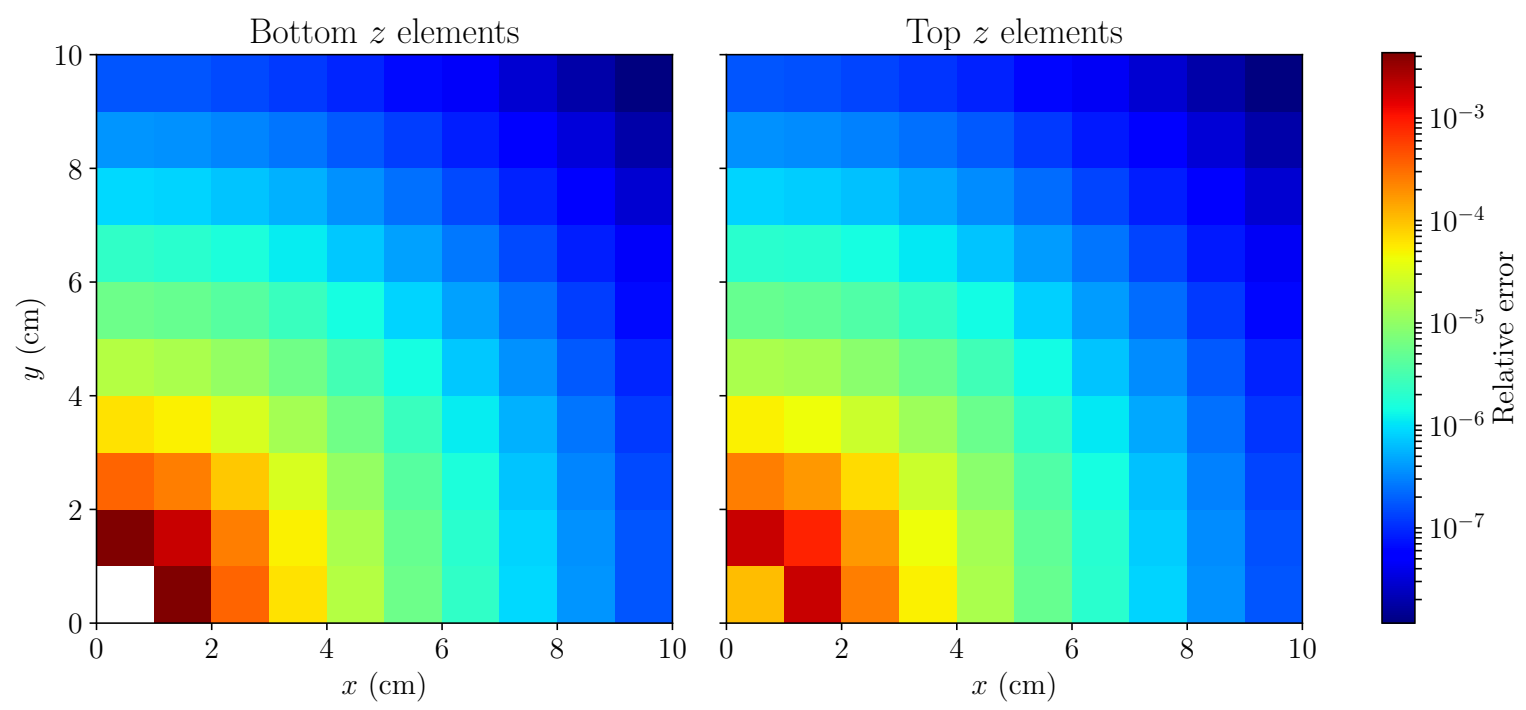

Figure 4: Comparison of relative errors in the integrated uncollided flux in each element in an absorbing medium $\left(\Sigma_{t}=0.5 \mathrm{~cm}\right)$ with 8 Gaussian quadrature points per element.

\subsection{Volumetric Source to Volumetric Targets}

A volumetric source that emits $1 \mathrm{n} / \mathrm{cm}^{3} \cdot \mathrm{s}$ is located in the element with corners $(0,0,0)$ and $(1,1,1)$ in a $10 \times 10 \times 2$ mesh of cuboid elements of width $1 \mathrm{~cm} \times 1 \mathrm{~cm} \times 1 \mathrm{~cm}$. The desired quantity again is the integrated uncollided flux in each element (the target elements) except for the element that contains the source (the element with lower vertex $(0,0,0)$ and upper vertex $(1,1,1))$. The total cross section of the targets and source element is then varied. The reference solution was obtained by using the ray-tracing implementation with 1000 Gaussian quadrature points per element and a uniform mesh refinement of 4 . The results for two of the target elements are as seen below in Table 2 and Figure 5. 
Table 2: Volumetric source to volumetric target results for the element defined by the corners $(4,0,0)$ and $(5,1,1)$ and the element defined by the corners $(8,0,0)$ and $(9,1,1)$.

\begin{tabular}{cccccc}
\hline \multirow{2}{*}{ Medium } & $\begin{array}{c}\text { Quad pts. } \\
\text { (1D) }\end{array}$ & Solution & Relative error & Solution & Relative error \\
\hline \multirow{5}{*}{ Void } & 1 & $4.9735919716 \times 10^{-3}$ & $1.0181726448 \times 10^{-2}$ & $1.2433979929 \times 10^{-3}$ & $2.5892932111 \times 10^{-3}$ \\
& 2 & $5.0232227257 \times 10^{-3}$ & $3.0447322665 \times 10^{-4}$ & $1.2466021624 \times 10^{-3}$ & $1.9019678231 \times 10^{-5}$ \\
& 3 & $5.0247487574 \times 10^{-3}$ & $7.7038059141 \times 10^{-7}$ & $1.2466258563 \times 10^{-3}$ & $1.3229412905 \times 10^{-8}$ \\
& 4 & $5.0247525910 \times 10^{-3}$ & $7.4378389145 \times 10^{-9}$ & $1.2466258728 \times 10^{-3}$ & $2.9565073448 \times 10^{-11}$ \\
& 5 & $5.0247526283 \times 10^{-3}$ & $1.9902659579 \times 10^{-11}$ & $1.2466258728 \times 10^{-3}$ & $9.1493423194 \times 10^{-14}$ \\
\cline { 2 - 6 } & Reference & $5.0247526284 \times 10^{-3}$ & & $1.2466258728 \times 10^{-3}$ & \\
\hline \multirow{2}{*}{ Source } & 1 & $3.3338983994 \times 10^{-3}$ & $1.5059305153 \times 10^{-2}$ & $5.5869473214 \times 10^{-4}$ & $5.4848549938 \times 10^{-3}$ \\
$\Sigma_{t}^{s}=0.1 \mathrm{~cm}$ & 2 & $3.3834902226 \times 10^{-3}$ & $4.0828733986 \times 10^{-4}$ & $5.6175692013 \times 10^{-4}$ & $3.3949236368 \times 10^{-5}$ \\
& 3 & $3.3848684216 \times 10^{-3}$ & $1.1230851004 \times 10^{-6}$ & $5.6177597643 \times 10^{-4}$ & $2.7705646541 \times 10^{-8}$ \\
Target & 4 & $3.3848721877 \times 10^{-3}$ & $1.0461899537 \times 10^{-8}$ & $5.6177599196 \times 10^{-4}$ & $5.8622371655 \times 10^{-11}$ \\
$\Sigma_{t}^{t}=0.1 \mathrm{~cm}$ & 5 & $3.3848722230 \times 10^{-3}$ & $2.9212231746 \times 10^{-11}$ & $5.6177599199 \times 10^{-4}$ & $1.3779876004 \times 10^{-13}$ \\
\cline { 2 - 6 } & Reference & $3.3848722231 \times 10^{-3}$ & & $5.6177599199 \times 10^{-4}$ & \\
\hline \multirow{2}{*}{ Source } & 1 & $2.3493584941 \times 10^{-3}$ & $1.6671993197 \times 10^{-2}$ & $2.6390870463 \times 10^{-4}$ & $6.8330566361 \times 10^{-3}$ \\
$\Sigma_{t}^{s}=0.1 \mathrm{~cm}$ & 2 & $2.3880376191 \times 10^{-3}$ & $4.8277942236 \times 10^{-4}$ & $2.6571172491 \times 10^{-4}$ & $4.7755092326 \times 10^{-5}$ \\
& 3 & $2.3891879848 \times 10^{-3}$ & $1.2919325826 \times 10^{-6}$ & $2.6572440409 \times 10^{-4}$ & $3.9567742807 \times 10^{-8}$ \\
Target & 4 & $2.3891910414 \times 10^{-3}$ & $1.2554813836 \times 10^{-8}$ & $2.6572441458 \times 10^{-4}$ & $8.8442898168 \times 10^{-11}$ \\
$\Sigma_{t}^{t}=0.2 \mathrm{~cm}$ & 5 & $2.3891910714 \times 10^{-3}$ & $3.3097241939 \times 10^{-11}$ & $2.6572441460 \times 10^{-4}$ & $1.5096648323 \times 10^{-13}$ \\
\cline { 2 - 6 } & Reference & $2.3891910714 \times 10^{-3}$ & & $2.6572441460 \times 10^{-4}$ & \\
\hline
\end{tabular}

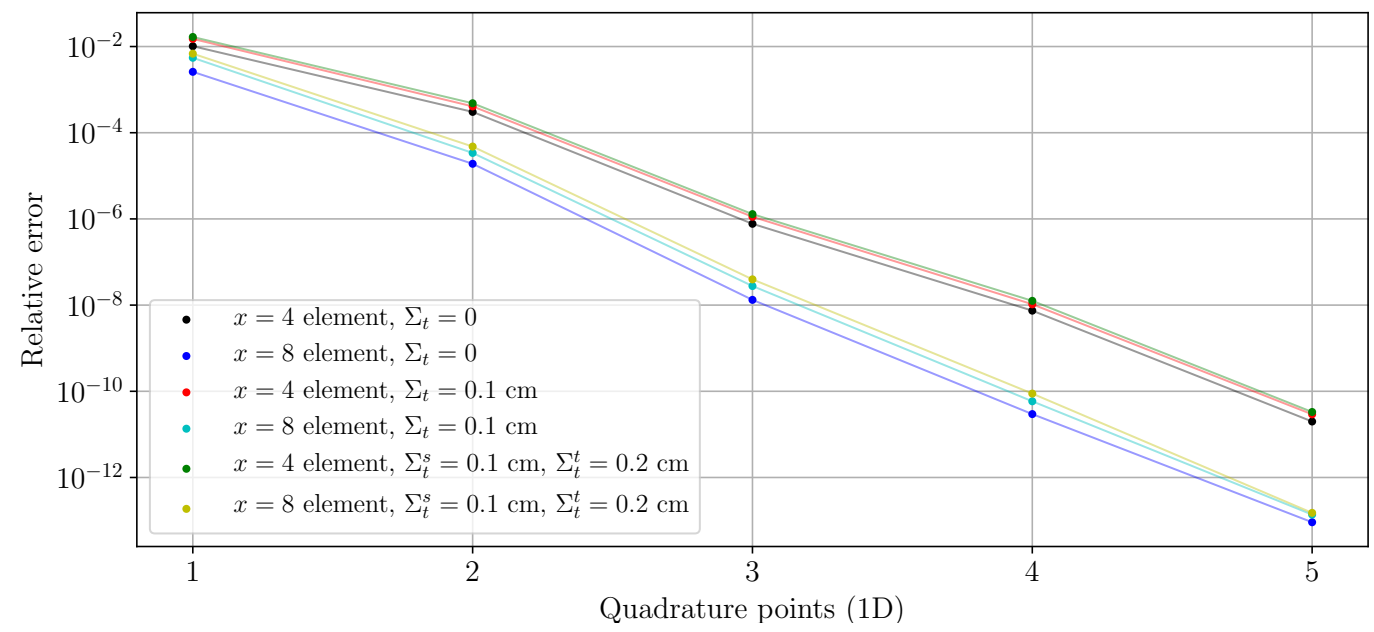

Figure 5: Comparison of relative errors in the integrated uncollided flux for two elements with a volumetric source and target. Element $x=4$ is the element defined by the corners $(4,0,0)$ and $(5,1,1)$ and element $x=8$ is the element defined by the corners $(8,0,0)$ and $(9,1,1) . \Sigma_{t}^{s}$ is the source cross section and $\Sigma_{t}^{t}$ is the target cross section. 


\subsection{Kobayashi Benchmark}

The Kobayashi 3-D benchmark void problems proposed by Kobayashi [7] consist of two sets of one-group source problems with similar geometry. The first set of problems only contains void and a pure absorbing medium, while the other replaces the pure absorber with a material which has a scattering cross-section of $50 \%$ of the total-cross section. In particular, the pure absorbing case of problem ii was chosen as a visual verification for the implementation (and more complex test) thus far. The geometry is as follows in Figure 6. The total cross sections for regions 1,2 , and 3 are $0.1 \mathrm{~cm}^{-1}, 10^{-4} \mathrm{~cm}$, and $0.1 \mathrm{~cm}^{-1}$, respectively. The source strength is $1.0 \mathrm{n} / \mathrm{cm}^{3} \cdot \mathrm{s}$.

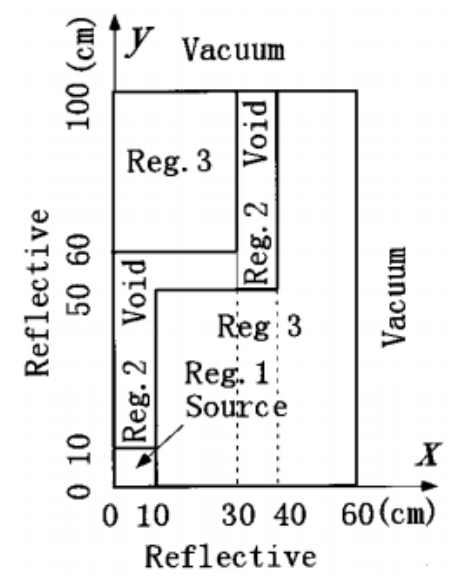

(a) $x-y$ plane

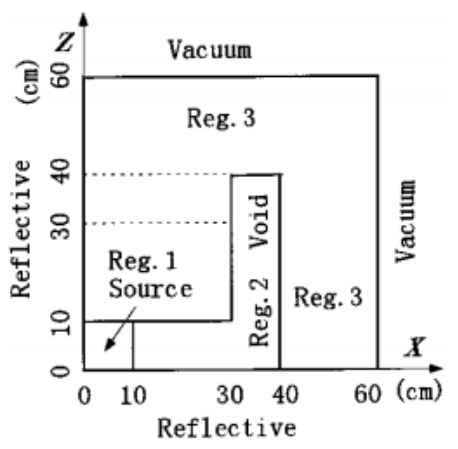

(b) $x-z$ plane

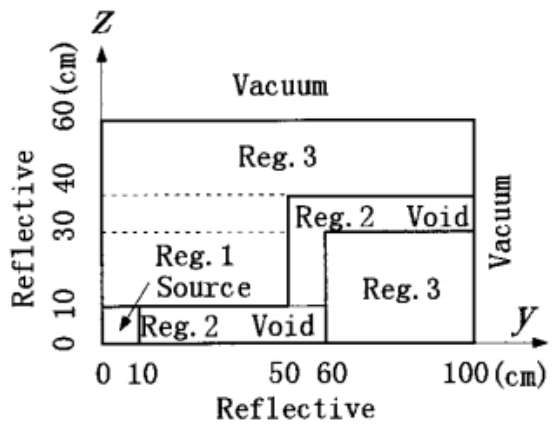

(c) $y-z$ plane

Figure 6: The geometry for the Kobayashi benchmark problem ii. [7]

As the ray-tracing implementation does not currently support reflecting boundary conditions, one set of the source elements was mirrored across each reflective boundary in the input file. Due to this, the axes seen above in the benchmark specifications are translated by $10 \mathrm{~cm}$ in each direction for each of the result plots below. The results were obtained in a mesh of elements $5 \mathrm{~cm} \times 5 \mathrm{~cm} \times 5 \mathrm{~cm}$. Rays were traced from source elements that contained 343 Gaussian quadrature points each, to target element centroids. 


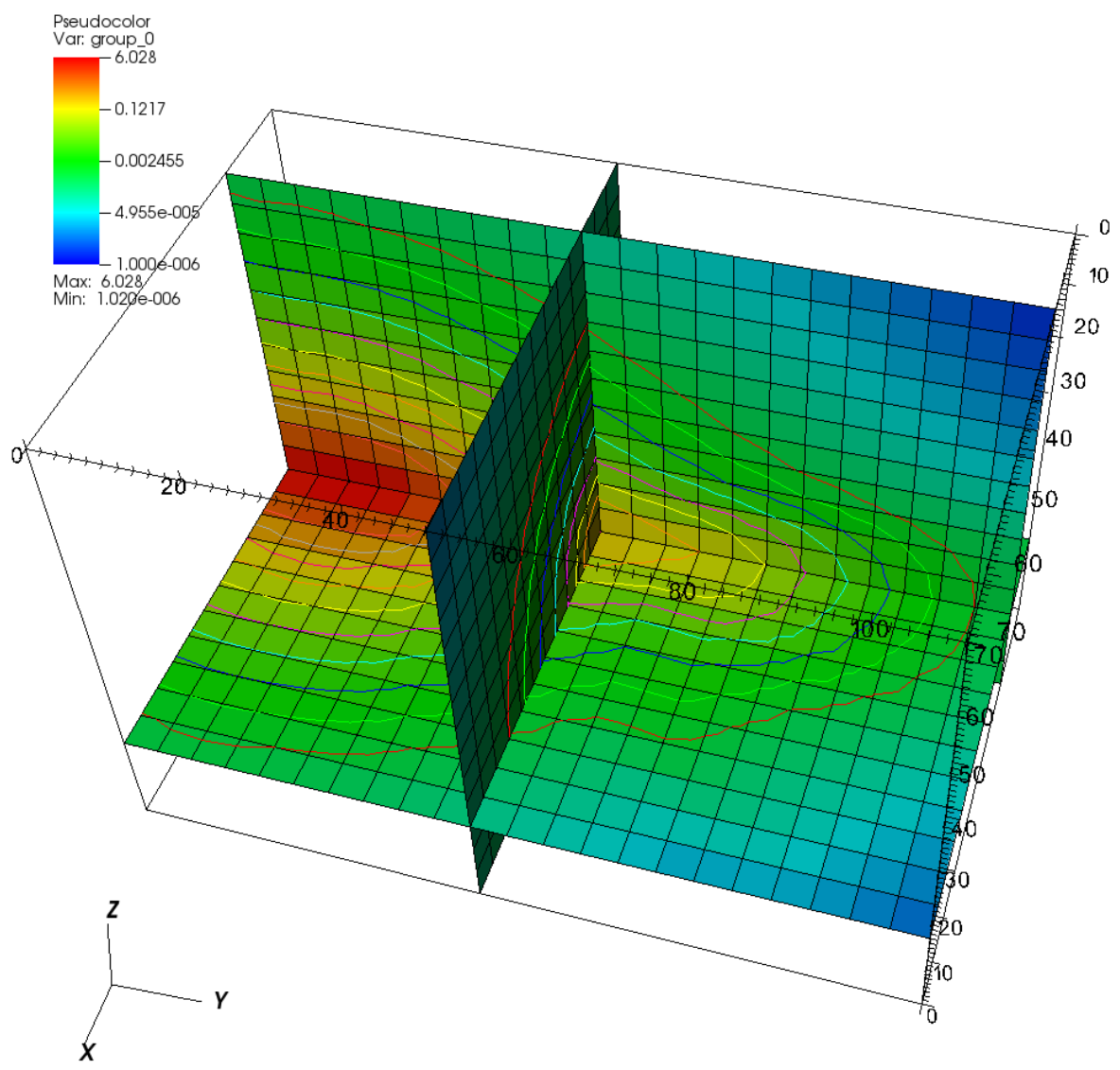

Figure 7: The cell-averaged uncollided flux result for the Kobayashi benchmark problem ii in a pure absorber. Viewed is the duct extending directly from the source elements. The contour lines are evenly spaced between 1 and $10^{-3}$ (log scale). The axes are shifted 10 $\mathrm{cm}$ in every direction compared to the benchmark geometries in order to account for the reflecting boundaries. 


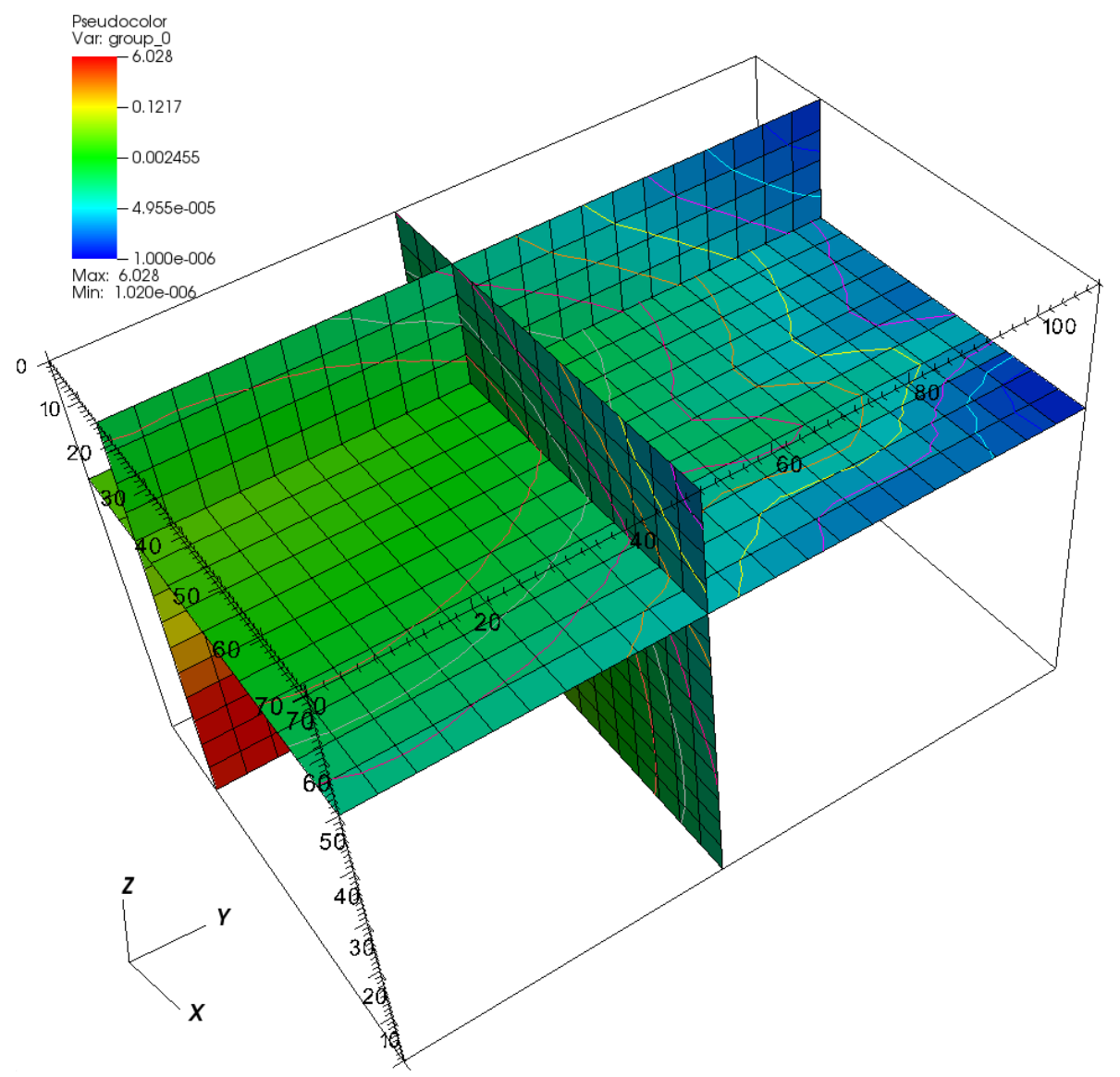

Figure 8: The cell-averaged uncollided flux result for the Kobayashi benchmark problem ii in a pure absorber. Viewed is the top duct plane extending towards the rear $y-z$ face. The contour lines are evenly spaced between $10^{-4}$ and $10^{-6}$ (log scale). The axes are shifted $10 \mathrm{~cm}$ in every direction compared to the benchmark geometries in order to account for the reflecting boundaries. 


\section{Future Work}

The longer-term goal of this research is to utilize this implementation for both first- and lastcollided source calculations in Rattlesnake. An initial implementation has been completed thus far and the future goals are as follows.

\subsection{Ray-Tracing Functionality}

\subsubsection{Tracing to target surfaces and points}

There exist cases where an exiting flux is desired on a surface, or a where point flux value is desired. The ray_tracing module supports only constant monomial values on each element at the time of this report. The theory behind tracing instead to points and surfaces is similar to the theory already discussed above. The significant issue lies in the ray_tracing expansion beyond constant monomial values and a clean implementation in MOOSE in which is able to store data into element quadrature.

\subsubsection{Reflective boundary conditions}

The ray_tracing module currently supports reflective boundary conditions, but the primary task is spawning rays in the directions such that they will reflect to the desired target points. For each reflective boundary, each target point is mirrored across the boundary. For each source point, a ray is then spawned towards the mirrored image of each target point and the ray_tracing module handles the reflection appropriately. If there is more than one reflected boundary, target points will be mirrored multiple times in order to determine the twice, third, etc., reflected source contribution at each target point. In cases where there is a potential for infinite reflection, the ray is to be killed after it has traveled a sufficient distance that its source contribution is minimal. 


\subsection{Methods development}

\subsubsection{First and last-collided Source}

The current implementation is a standalone capability, which takes as input a set of target elements and source elements and returns the uncollided flux solution. In order for the ray-tracer to be used in Rattlesnake calculations, it is necessary to implement it into the action system in a manner that the user would enable a first and last-collided source option. Through the action system, the ray-tracer will be executed and will make available the mesh-wide uncollided flux as necessary for the transport calculation (as a first and lastcollided source).

\subsubsection{Spatial and angular moments}

The current implementation returns only cell-averaged uncollided fluxes. As a result of this, the only method to obtain a refined source for use in a transport calculation is to refine the ray-tracing mesh and then superimpose the ray-tracing solution on a coarser mesh for use in the $S_{N}$ calculation. This is potentially costly due to the increased number of segments required for each generated ray.

With materials whose scattering cross sections are not isotropic, the zeroth angular moment of the uncollided flux is not sufficient for use in any collided source calculations. There is also desire for the angular flux solution on a surface, which would require higher moments.

Both implementations are straight-forward and the theory is already written for this implementation. The data carried by each traversed ray is user-set and scaled as necessary. 


\subsection{Conclusions}

With TREAT restart imminent, likely as soon as November 2017, modeling and simulation efforts at INL have focused on calculation of steady-state fluxes and time-dependent fluxes with thermal feedback within the core using Rattlesnake along with BISON in MAMMOTH. Such capabilities will be essential for initial simulation of transient core behavior, initially to be used for experiment design and interpretation of experimental measurements. Startup measurements will be performed in early 2018 to be used to validate these methods. Early measurements will not use the hodoscope system for fuel monitoring.

However, as TREAT experimental measurement capabilities mature in the years following restart, experiment visualization efforts will also resume using the fast-neutron hodoscope. Simulation of neutron transport between a target fuel sample and the hodoscope detectors will allow improvement of experiment design to maximize hodoscope imagery. In addition, efforts are underway to replace the current hodoscope with a modern high-resolution system. Design calculations currently are using Monte Carlo methods, requiring significant computational effort while still yielding large statistical uncertainties, challenging design efforts.

The work described in this report is the first stage of a longer-term research/development project to provide an efficient and accurate method for solution of the transport equation that will account for the strongly anisotropic radiation transport through hodoscope collimator ducts. We have derived the method to compute the uncollided flux for any target cells within a 3-D finite element meshm and successfully applied Gaston's ray tracing algorithm within Rattlesnake to lay the groundwork needed to develop the ability to determine the element-average uncollided fluxes as a result of a set of point sources and volumetric sources. The Kobayashi set of 3-D benchmark void problems has been used to demonstrate the viability of the approach. Ultimately, the methods developed here will be incorporated within Rattlesnake as an action system in such a manner that a Rattlesnake user would enable a first and last-collided source option to aid in hodoscope calculations. And although the TREAT hodoscope is the motivation for this work, the methods developed here

will aid in deep penetration streaming calculations in other neutron transport problems, as demonstrated in the Kobayashi calculations. 


\section{References}

[1] U.S. Department of Energy, Mission Need Statement for the Resumption of Transient Fuel Testing, U.S. DOE, December 3, 2010.

[2] U.S. Department of Energy, Resumption of Transient Testing Capability, April 15, 2013, http://energy.gov/ne/articles/resumption-transient-testing

[3] G. A. Fruend, H. P. Iskendarian and D. Okrent, TREAT, a Pulsed Graphite- Moderated Reactor for Kinetics Experiments, Proc. 2nd United Nations Int. Conf. on the Peaceful Uses of Atomic Energy, Geneva, Switzerland, 10, 461-475 (1958).

[4] Javier Ortensi, Mark D. DeHart, Frederick N. Gleicher, Yaqi Wang, and Sebastian Schunert, "Full Core TREAT Kinetics Demonstration Using Rattlesnake/BISON Coupling Within MAMMOTH," INL/EXT-15-36268, Idaho National Laboratory, August 2015.

[5] Javier Ortensi, Benjamin Baker, Yaqi Wang, Sebastian Schunert and Mark DeHart, "Transient Simulation of the Multi-SERTTA Experiment with MAMMOTH," INL/EXT-17-42404, Idaho National Laboratory, June 2017.

[6] D. L. Chichester, S. M. Watson, J. T. Johnson, and D. M. Wachs, "The TREAT FastNeutron Hodoscope and Plans for Restoring it to Operation," INL/CON-15-34260, Idaho National Laboratory.

[7] K. Kobayashi, A Proposal for 3-D Radiation Transport Benchmarks for Simple Geometries with Void Region, 3-D Deterministic Radiation Transport Computer Programs, OECD Proceedings, p. 403 (1997). 
